\title{
Article
}

\section{Effect of cadence selection on peak power and time of power production in elite BMX riders; a laboratory based study.}

Bentley, Ian, Hurst, Howard Thomas, Rylands, Lee and Roberts, Simon

Available at http://clok.uclan.ac.uk/15458/

Bentley, Ian ORCID: 0000-0002-9086-2338, Hurst, Howard Thomas ORCID: 0000-0001-7889-8592, Rylands, Lee and Roberts, Simon (2016) Effect of cadence selection on peak power and time of power production in elite BMX riders; a laboratory based study. Journal of Sports Sciences . ISSN 0264-0414

It is advisable to refer to the publisher's version if you intend to cite from the work. http://dx.doi.org/10.1080/02640414.2016.1215491

For more information about UCLan's research in this area go to http://www.uclan.ac.uk/researchgroups/ and search for <name of research Group>.

For information about Research generally at UCLan please go to http://www.uclan.ac.uk/research/

All outputs in CLoK are protected by Intellectual Property Rights law, including Copyright law. Copyright, IPR and Moral Rights for the works on this site are retained by the individual authors and/or other copyright owners. Terms and conditions for use of this material are defined in the policies page. 


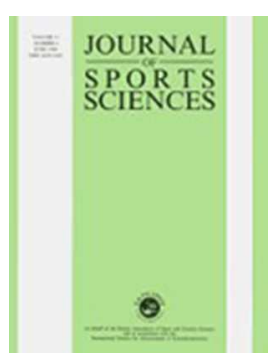

\section{Effect of cadence selection on peak power and time of power production in elite BMX riders; a laboratory based study.}

\begin{tabular}{|r|l|}
\hline Journal: & Journal of Sports Sciences \\
\hline Manuscript ID & RJSP-2014-0838.R4 \\
\hline Manuscript Type: & Special Issue \\
\hline Keywords: & Bicycle motocross, peak power, cadence, time to peak power \\
\hline \multicolumn{2}{|c}{} \\
\hline
\end{tabular}

SCHOLARONE $^{\text {th }}$

Manuscripts 
1

2

3

4

5

6

7

8

9

10

11

12

13

14

15

16

17

18

19

20

21

22

23

24

25

26

27

28

29

30

31

32

33

34

35

36

37

38

39

40

41

42

43

44

45

46

47

48

49

50

51

52

53

54

55

56

57

58

59

60

Effect of cadence selection on peak power and time of power production in elite BMX riders: a laboratory based study. 


\begin{abstract}
The aims of this study were to analyse the optimal cadence for peak power production and time to peak power in bicycle motocross (BMX) riders. Six male elite BMX riders volunteered for the study. Each rider completed 3 maximal sprints at a cadence of $80,100,120$ and 140 revs $\cdot \mathrm{min}^{-1}$ on a laboratory Schoberer Rad Messtechnik (SRM) cycle ergometer in isokinetic mode. The riders' mean values for peak power and time of power production in all three tests were recorded. The BMX riders produced peak power $(1105 \pm 139 \mathrm{~W})$ at $100 \mathrm{revs} \cdot \mathrm{min}^{-1}$ with lower peak power produced at $80 \mathrm{revs} \cdot \mathrm{min}^{-1} \quad\left(1060 \pm 69 \mathrm{~W}, \quad\left(F(2,15)=3.162 ; \quad p=.266 ; \quad \eta^{2}=0.960\right)\right.$, 120revs $\cdot \min ^{-1}\left(1077 \pm 141 \mathrm{~W},\left(F(2,15)=4.348 ; p=.203 ; \eta^{2}=0.970\right)\right.$ and 140revs $\cdot \mathrm{min}^{-1}$ $\left(1046 \pm 175 \mathrm{~W},\left(F(2,15)=12.350 ; p=0.077 ; \eta^{2}=0.989\right)\right.$. The shortest time to power production was attained at $120 \mathrm{revs} \cdot \mathrm{min}^{-1}$ in $2.5 \pm 1.07 \mathrm{~s}$. Whilst a cadence of 80revs $\cdot \min ^{-1} \quad\left(3.5 \pm 0.8 s, \quad\left(F(2,15)=2.667 ; \quad p=.284 ; \quad \eta^{2}=0.800\right) \quad\right.$ 100revs $\cdot \min ^{-1}$ (3.00 $\pm 1.13 \mathrm{~s}, \quad\left(F(2,15)=24.832 ; p=.039 ; \eta^{2}=0.974\right)$ and $140 \mathrm{revs} \cdot \mathrm{min}^{-1}(3.50 \pm 0.88 \mathrm{~s}$, $\left.\left(F(2,15)=44.167 ; p=.006 ; \eta^{2}=0.967\right)\right)$ all recorded a longer time to peak power production. The results indicate that the optimal cadence for producing peak power output and reducing the time to peak power output are attained at comparatively low cadences for sprint cycling events. These findings could potentially inform strength and conditioning training to maximise dynamic force production and enable coaches to select optimal gear ratios.
\end{abstract}

\title{
Keywords: Time to peak power, cadence, peak power, bicycle motocross.
}




\section{Introduction}

The sport of bicycle motocross (BMX) is a sprint type event (Zabala et al., 2008) typically contested between eight riders over a track distance ranging between $300 m-400 m$. In contrast to other cycling disciplines (i.e. road cycling and mountain bike racing) the duration of a BMX race is short (35-45 seconds). Due to the short duration of a typical BMX race, and the reported difficulties with over-taking (Debraux et al., 2013), much of the scientific BMX has been dedicated to the importance of the start (Mateo et al., 2011; Zabala et al., 2009). The significance of the start was recently confirmed through a secondary analysis of the 2012 UCI BMX World Cup series data (Rylands \& Roberts, 2014). In this study the authors demonstrated a large correlation existed between the riders' position 8-10 seconds into a race and their eventual finish line placing. Despite previous research suggesting peak power is attained in the first $6 \mathrm{~m}$ of the race (Herman et al., 2009), Rylands and Roberts (2014) were unable to establish whether a relative physiological relationship existed between power production and pedalling rate (cadence). Consequently it has been suggested that future BMX research should focus on power production and at the start of the race (Herman, 2009).

The influence of torque, power and pedal rate on cycling performance has been well documented in a number of other cycling disciplines. For instance, using twelve elite sprint cyclists Dorel et al. (2005) demonstrated the importance of the power and cadence relationship in velodrome track sprint events. Dorel et al. (2005) concluded that the optimisation of the ratio between peak power and optimal cadence 
represents a key factor in track cycling performance. The authors found an optimal ratio during competition resulted in peak powers of $1600 \pm 116 \mathrm{~W}$ at a cadence of $129.8 \pm 4.7$ revs $\cdot \mathrm{min}^{-1}$. Similarly Abbiss et al. (2009) found that peak power was associated with higher pedal cadences of $100-120$ revs $\cdot \mathrm{min}^{-1}$ for track sprint cyclists. Moreover, endurance cycling events of $<4 \mathrm{~h}$ report power/cadence relationships of $90-100$ revs $\cdot \mathrm{min}^{-1}$ resulting in an optimal performance outcome, whereas cycling events $>4 \mathrm{~h}$ recorded an optimal relationship at $70-90$ revs $\cdot \mathrm{min}^{-1}$ (Abbiss et al., 2009). Consequently leading Abbiss et al. (2009) to conclude that the relationship between optimal cadence and power is specific to each individual cycling discipline. To date, however, there is a shortage of BMX related research in this particular area. Thus it is currently unclear whether an optimal relationship exists between power and cadence in BMX racing.

Previous field-based BMX research has shown that peak power output (2087 $\pm 157 \mathrm{~W}$ ) occurred at a cadence of $212 \pm 3.5 \mathrm{revs} \cdot \mathrm{min}^{-1}$ (Herman et al., 2009). Gardner et al. (2007) ascertained that peak power $(1791 \pm 169 \mathrm{~W})$ occurred at much lower cadences $\left(128 \pm 7 \mathrm{revs} \cdot \mathrm{min}^{-1}\right)$ in velodrome track sprint cyclists. Rylands et al. (2013) also reported peak power $(1256 \pm 276 \mathrm{~W})$ occurred at much lower cadences $\left(110 \pm 11\right.$ revs $\left.\cdot \mathrm{min}^{-1}\right)$ in BMX riders. The findings of Rylands and Roberts (2013) are comparable to those of Hurst et al. (2012) who reported peak power $(1138 \pm 136)$ occurred at a cadence of $115 \pm 5 \mathrm{revs} \cdot \mathrm{min}^{-1}$ in elite Downhill mountain bike (MTB) cyclists. Like BMX, Downhill MTB also requires competitors to accelerate from a start gate/hut down a ramp and thus may explain some of the similarities between these studies. 
However, to date the only study which has examined the optimal relationship between power and pedalling rate in BMX riders was conducted by Debraux and Bertucci (2011). Using a PowerTap hub-based powermeter system (Professional model, CycleOps, USA) and photoelectric cells, seven elite riders performed three, 80m sprints on a flat asphalt surface. Debraux and Bertucci (2011) analysed peak power and cadence produced during the $80 \mathrm{~m}$ sprint test. The data formed an inverted u shaped parabolic graph. This graph was used to extrapolate an optimal theoretical cadence that elicited peak power $\left(122 \pm 18 \mathrm{revs} \cdot \mathrm{min}^{-1}\right)$. Previous studies in this area (Sergeant et al., 1981; Vandewalle et al., 1985; Martin et al., 2007), albeit in other cycling disciplines, suggested that using a single data set to identify optimal relationships cannot be used to accurately ascertain the relationship. Subsequently, the actual optimal cadence to elicit peak power in BMX cyclists may be different from that reported by Debraux and Bertucci (2011).

The purpose of this study was to investigate the nature of any relationship between optimal power production and cadence in elite BMX riders and gain actual rather than predictive data. Due to a shortage of standardised testing protocols a broad range of cadences were employed (i.e. 80, 100, 120 and 140revs $\cdot \mathrm{min}^{-1}$ ).

\section{Methods}

\section{Participants}

Seventeen riders in the United Kingdom (UK) currently hold an elite level licence and from this population six elite male BMX riders volunteered to participate in the study. Mean rider characteristics were age $20 \pm 2 \mathrm{yrs}$, stature $169.0 \pm 5.0 \mathrm{~cm}$, body mass $68 \pm 4 \mathrm{~kg}$ and body fat $10 \pm 3 \%$. Stature was recorded using a Harpenden stadiometer (Cranlea, UK) to the nearest $0.1 \mathrm{~cm}$, whilst body mass and body fat were determined 
using air displacement plethysmography (Bod Pod, Life Systems International, USA). All participants had previous experiences of using laboratory cycle ergometers in isokinetic mode as adopted in the current study. Written and informed consent was obtained from participants and a detailed description of the test protocol was issued to them prior to the study. The study was approved by the University of Derby's Ethics Human Studies Board and in accordance with the Declaration of Helsinki.

\section{Experimental Design}

The relationship between power and cadence in elite BMX cycling was determined by each subject performing three repeated sprint tests on a Laboratory Schoberer Rad Messtechnik (SRM) 8 strange gauge cycle ergometer in isokinetic mode. The ergometer was configured and adapted to more accurately resemble a BMX bike used in competition. This was achieved through the fitting of a standard $70 \mathrm{~cm}$ straight bar to the ergometer, along with Shimano SPD pedals, and a crank length of $175 \mathrm{~cm}$. The bar was individually adjusted by each rider to their preferred height prior to the test. The saddle was lowered so it did not interfere with the rider when performing each sprint. An inertial load of $50.2 \mathrm{~kg} \cdot \mathrm{m}^{2}$ was added to the cycle ergometer which according to Debraux et al. (2013) equates to a standard BMX gear ratio of 43 (front chain ring)/16 (rear chain ring). Sampling frequency was set to $2 \mathrm{~Hz}$.

Prior to administration of the testing protocol each rider performed a structured selfpaced warm up in a seated position, and a series of short standing sprints. Each participant then performed three 10-second sprints in a standing position and was encouraged to reach maximal power in the shortest possible duration. Following this, mean time of the three sprints was calculated. Each rider performed the sprints from 
a stationary position with their self-selected leg in the lead position. Ten-minute rest periods were provided in between tests and then the riders repeated the test protocol at a different cadence. The three sprints were performed at cadences of $80,100,120$ and 140 revs $\cdot \min ^{-1}$. The rider's absolute values for peak power and time to peak power were recorded. The cadence was electromagnetically restricted by the SRM cycle ergometer. The cadences were randomly assigned and the riders were not informed of the cadence selection prior or during the test. Data from all six riders' sprints were recorded using SRMWin software version 5.

\section{Statistical analyses}

Data was initially screened for missing and implausible values and assumptions for normality, linearity and homogeneity of variances were conducted. The independent variable in the analysis was cadence $\left(80,100,120,140\right.$ revs $\left.\cdot \mathrm{min}^{-1}\right)$. Whilst the two dependant variables were peak power and time to peak power. Two within groups repeated measure analysis of variance (ANOVA) were used to determine significance. Where a significant difference was observed, Bonferroni pairwise comparisons were used to determine where the differences lay. Where sphericity was violated a Greenhouse-Geiser correction was used.

Due to the relatively small sample size, partial eta squared was used to determine the degree of total association between an effect and the dependent variable. Partial eta squared were interpreted based on their magnitude, where a value between 0.0 0.1 indicates a small effect, $0.1-0.3$ a medium effect, $0.3-0.5$ a moderate effect and $>0.5$ is a large effect (Tolmie et al., 2011). All statistical analyses were conducted using IBM Statistical Package for the Social Sciences statistical version 
19 (IBM inc., Chicago IL). For all statistical analysis, a $\mathrm{P}<0.05$ was accepted as the level of significance.

\section{Results}

The main area of interest in this study was whether cadence selection has an effect on peak power and time to peak power in elite BMX cyclists. Analysis of the within groups repeated measure ANOVA revealed no significant differences between the cadences $\left(80,100,120\right.$ and 140 revs $\left.\cdot \mathrm{min}^{-1}\right)$ for peak power $\left(F(1,17)=.799 ; p=.424 ; \eta^{2}\right.$ $=0.045)$, and time to peak power $\left(F(1,17)=.691 ; p=.532 ; \eta^{2}=0.121\right)$ between the selected cadences.

The riders' mean and individual results from the three sprints are highlighted in table 1, whilst figure one gives a comparative view of the riders' mean results.

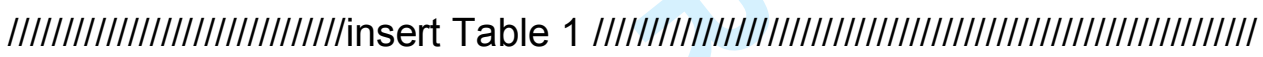

The optimal relationship between cadence and time to peak power production was achieved at a cadence of $120 \mathrm{revs} \cdot \mathrm{min}^{-1}$ in a time of $2.50 \pm 1.07 \mathrm{~s}$. The highest cadence of $140 \mathrm{revs} \cdot \mathrm{min}^{-1}$ and lowest cadence of $80 \mathrm{revs} \cdot \mathrm{min}^{-1}$ resulted in the greatest time to peak power production, $3.50 \pm 0.88 \mathrm{~s}, 3.50 \pm 0.80$ s respectively (see figure 1 ). A large effect size was also noted when comparing the dependant variable of time to peak power $\left(120 \mathrm{revs} \cdot \mathrm{min}^{-1}, 2.50 \pm 1.07 \mathrm{~s}\right)$ to effect variables of 80,100 and $140 \mathrm{revs} \cdot \mathrm{min}^{-}$ ${ }^{1}$ revs: $\quad 80$ revs $\cdot \min ^{-1} \quad\left(F(2,15)=2.667 ; \quad p=.284 ; \quad \eta^{2}=0.800\right), \quad$ 100revs $\cdot \min ^{-1}$ $\left(F(2,15)=24.832 ; p=.039 ; \eta^{2}=0.974\right)$, 140revs $\cdot \min ^{-1} \quad\left(F(2,15)=44.167 ; p=.006 ; \eta^{2}\right.$ $=0.967)$. The results demonstrate that a difference in time to peak power occurred when comparing them to the optimal cadence of $120 \mathrm{revs} \cdot \mathrm{min}^{-1}$. 
The optimal relationship between cadence and peak power occurred at a cadence of 100revs $\cdot \min ^{-1}(1105 \pm 139 \mathrm{~W})$, whilst the cadence of $140 \mathrm{revs} \cdot \mathrm{min}^{-1}$ resulted in the lowest peak power $(1046 \pm 175 \mathrm{~W})$ of all the cadences. Similar to the results found for time to peak power production, the peak power results demonstrate that there was a difference between the peak powers attained by the riders when restricted by each of the cadences. A large effect size was found between all the variable cadences $\left(80,120,140\right.$ revs $\left.\cdot \mathrm{min}^{-1}\right): \quad 80 \mathrm{revs} \cdot \mathrm{min}^{-1} \quad\left(F(2,15)=3.162 ; \quad \mathrm{p}=.266 ; \quad \eta^{2} \quad=0.960\right)$, 120revs $\cdot \min ^{-1}\left(F(2,15)=4.348 ; p=.203 ; \eta^{2}=0.970\right)$, 140revs $\cdot \min ^{-1} \quad(F(2,15)=12.350$; $\left.p=0.077 ; \eta^{2}=0.989\right)$.

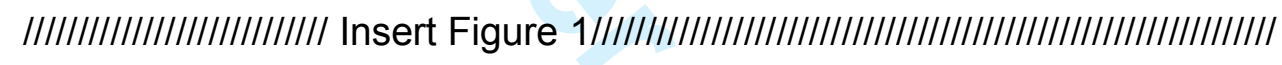

\section{Discussion}

The purpose of the current study was to determine the relationship between power and cadence in elite BMX racing through a controlled laboratory based study. The results show that peak power was achieved at a cadence of $100 \mathrm{revs} \cdot \mathrm{min}^{-1}$, whilst time to peak power was attained at a higher cadence of $120 \mathrm{revs} \cdot \mathrm{min}^{-1}$ (see figure 1 ). Therefore, a cadence range of between $100-120 \mathrm{revs} \cdot \mathrm{min}^{-1}$ was found to be the optimal for a BMX rider. Research has ascertained the optimal cadence range for other cycling disciplines (Abbiss et al., 2009. Dorel et al. 2005), but the current paper is the first to infer an optimal cadence for BMX cycling. The importance of these findings has implications for coaches and riders when selecting gear ratios for competition and training with the potential to affect future race outcomes.

Previous research has identified that cadence is one of the few variables a cyclist can adjust to manage performance and fatigue (Ansley \& Cangley, 2009). This is 
achieved by a rider consciously altering their cadence (i.e. leg speed) or mechanically through increasing or decreasing the bikes' gear ratio. Therefore, ascertaining the BMX rider's optimal cadence that enables optimal power generation is crucial in the selection of a gear ratio. Gardener et al. (2007) analysed the power cadence relationship in elite velodrome track sprinters and found the peak power occurred at $212 \pm 3.5 \mathrm{revs} \cdot \mathrm{min}^{-1}$. It could therefore be assumed, as BMX cycling is also a sprint type event, that the cadence power values would be similar. However, as these results demonstrate, peak power outputs were produced at a lower cadence than previously reported for other sprint cycling disciplines. Based on the findings in this study the authors agree with Abiss et al (2009) that optimal cadence power relationship is cycling discipline specific.

One of the few studies to examine the cadence power relationship in BMX riders suggested that maximal power was produced at relatively low cadences (i.e. 122 \pm 18 revs $\bullet \mathrm{min}^{-1}$. Debraux, 2011) for a sprint event. One possible explanation for the variation in Debraux's findings may be due to research design and data collection procedures. For instance Debraux (2011) extrapolated the optimal power cadence relationship, rather than directly measuring the relationship in five male and two female BMX riders. Furthermore, Debraux (2011) reported that an increase in load would result in an increase in peak power. The present study found the opposite occurred as the highest load of $80 \mathrm{revs} \cdot \mathrm{min}^{-1}$ produced the second lowest peak power $(1060 \pm 69 \mathrm{~W})$ of all the cadences tested (see table 1). This finding in particular may influence the choice of gear selection for a BMX rider. The implications of these findings suggest any decision to employ a high gear ratio, which in turn increases the load, does not appear to have an optimal impact on peak power. However, the actual 
gear ratios above and below a standard $43 / 16$ would still need to be verified. Whilst these findings have been established in a laboratory environment, further research in a BMX track environment is worthy of consideration.

The second finding of this study was the relationship between cadence and time of power production. The difference in the time of power production between the cadences (see table 1) may not appear to be significant. However, the application of these values when compared to actual race data reveals the importance of the findings and optimal cadence. According to publically accessible data (www.bmxresults.com) the variation in finish line times for elite level BMX races are marginal. Data from 175 races in 4 countries during the 2012 World Cup Supercross Series revealed a mean deviation in finish position of $1^{\text {st }}$ to second as $0.49 \pm 0.36 \mathrm{~s}$ and from $1^{\text {st }}$ to third place as $0.95 \pm 0.57 \mathrm{~s}$. The present study ascertained that optimal cadence selection can result in a 1.0 s decrease in time of power production. Based on the 2012 race data and the present study the implications for a rider could be the difference between placing $1^{\text {st }}$ or $4^{\text {th }}$. Furthermore, the current study utilised a 10 second sprint, whilst the data from the 2012 World Cup Series was based on the whole race lasting $32.16 \pm 3.12$ s (Rylands \& Roberts, 2013). As a result this finding could have a greater effect on overall finish placing's, not just for podium placed riders, but potentially also for lower ranked riders attaining a podium position.

\section{Conclusion}

The present study has contributed new research in an area short of empirical research by investigating the relationship between cadence and power in elite BMX racing. The study can provide a reference for the minimum level requirements 
expected for national standard riders. The study suggested that data from other cycling disciplines cannot be applied to the sport of BMX cycling as it is unique to that cycling discipline. The main findings from this study are the effect of cadence on peak power and time of power production. The results from the study could inform strength and conditioning training to elicit maximal dynamic force of the lower limbs of BMX riders and help inform gear selection.

\begin{abstract}
A potential limitation of the current study was the sampling rate. The minimum sampling rate of the SRM isokinetic ergometer was $2 \mathrm{hz}$. Alternative testing equipment could have been used and a greater data set recorded. However, few powermeters currently allow the accurate assessment of field-based torque analysis.
\end{abstract}

The number of riders tested in the study appears to be low and could be seen as a limitation of the study. However, in the UK at the time of data collection only 17 elite male BMX riders held a British Cycling Federation elite race licence. Therefore, the sample size used in this study constitutes $65 \%$ of the elite BMX population in the UK. To further this line of study it is recommended that future BMX studies look at the effect of gear selection on peak power and time to optimal power in the BMX track environment.

\title{
Acknowledgments
}

The authors gratefully acknowledge the British Cycling Federation Coach Jeremy Hayes for his time and dedication to the research project. 


\section{Reference}

Abbiss, C. R., Peiffer, J. J., Laursen, P. B. (2009). Optimal cadence selection during cycling. International Sport Medicine Journal, 10(1), 1-15.

Ansley, L., Cangley, P. (2009). Determinants of "optimal" cadence during cycling. European Journal of Sport Science, 9(2), 61-85.

Bertucci, W. M., Hourde, C. (2011). Laboratory testing and field performance in BMX riders. Journal of Sports Science and Medicine, 10(2), 417-419.

Craig, N. P., Norton, K. I. (2001). Characteristics of a track cyclist. Sports Medicine, $37(7), 457-468$.

Cohen, J. (1962). The statistical power of abnormal-social psychological research: a review. Journal of Abnormal and Social Psychology, 65(3), 145-153.

Dorel, S., Hautier, C. A., Rambaud, D., Van Praagh, E., Lacour, J. R., Bourdin, M. (2005).Torque and Power-Velocity Relationships in Cycling: Relevance to Track Sprint Performance in World-Class Cyclists. International Journal of Sports Medicine, 26(9), 739-746.

Debraux, P. Bertucci, W. (2011). Determining factors of the sprint performance in high-level BMX riders. Computer Methods in Biomechanics and Biomedical Engineering, 14(1), 53-55.

Debraux, P., Manolova, A. V., Soudain-Pineau, M., Hourde, C., Bertucci, W. (2013). Maximal torque and power pedaling rate relationships for high level BMX riders in field tests. Journal of Science in Cycling, 2(1), 51-57.

Gardner, S. A., Martin, J. C., Martin, D. T., Barras, M., Jenkins, D.G. (2007). Maximal torque- and power-pedaling rate relationships for elite sprint cyclists in laboratory and field tests. European Journal of Applied Physiology, 101(3), 287-292.

Herman, C. W., McGregor, S. J., Allen, H., Bollt, E. M. (2009). Power Capabilities of Elite Bicycle Motocross Racers During Field Testing In Preparation For 2008 Olympics. Medicine \& Science in Sports \& Exercise, 41(5), 306-307.

Hurst, H. T., Swarén, M., Hébert-Losier, K., Ericsson, F., Holmberg, H-C. (2012) Anaerobic Power and Cadence Characteristics of Elite Cross-Country and Downhill Mountain Bikers. Proceedings of the 17th Annual Congress of the European College of Sports Sciences, (Bruge, July 2012).

Mateo, M., Blasco-Lafarga, C., Zabala, M., Herna'ndez, M. (2011). Pedalling power and speed production Vs. Technical factors and track difficulty in bicycle motor cross cycling. Journal of Strength and Conditioning Research, 25(12), 3248-3256. 
Rylands, L. Roberts, S. J., Cheetham, M., Baker, A. (2014). Velocity production in elite BMX Riders: A field based Study Using SRM power meter. Journal of Exercise Physiology, 16(3), 14-23.

Tolmie, A., Muijs, D., \& McAteer, E. (2011). Quantitative methods in educational and social research using SPSS. Berkshire, England: Open University Press.

Sargeant, A. J., Hoinville, E., Young, A. (1981). Maximal leg force and power output during short term dynamic exercises. Journal of Applied Physiology, 51(5), 11751182.

Zabala, M., Requena, B., Sanchez-munoz, C., Gonzalez-Badillo, J., Garcia, I., Oopik, V., Paasuke, M. (2008). Effects of sodium bicarbonate ingestion on performance and perceptual responses in a laboratory-simulated BMX cycling qualification series. Journal of Strength and Conditioning research, 22(5), 16461653.

Zabala, M., Sánchez-Muñoz, C., Mateo, M. (2009). Effects of the administration of feedback on performance of the BMX cycling gate start. Journal of Sports Science and Medicine, 8(3), 393-400.

Vandewalle, H. G., Heller, J. P., Monod, H. (1985). All out anaerobic capacity tests on cycle ergometers: a comparative study on men and women. European Journal of Applied Physiology, 54(2), 222-229. 
Table 1

80revs $\cdot \min ^{-1} \quad$ 100revs $\cdot \min ^{-1} \quad$ 120revs $\cdot \min ^{-1} \quad$ 140revs $\cdot \mathrm{min}^{-1}$

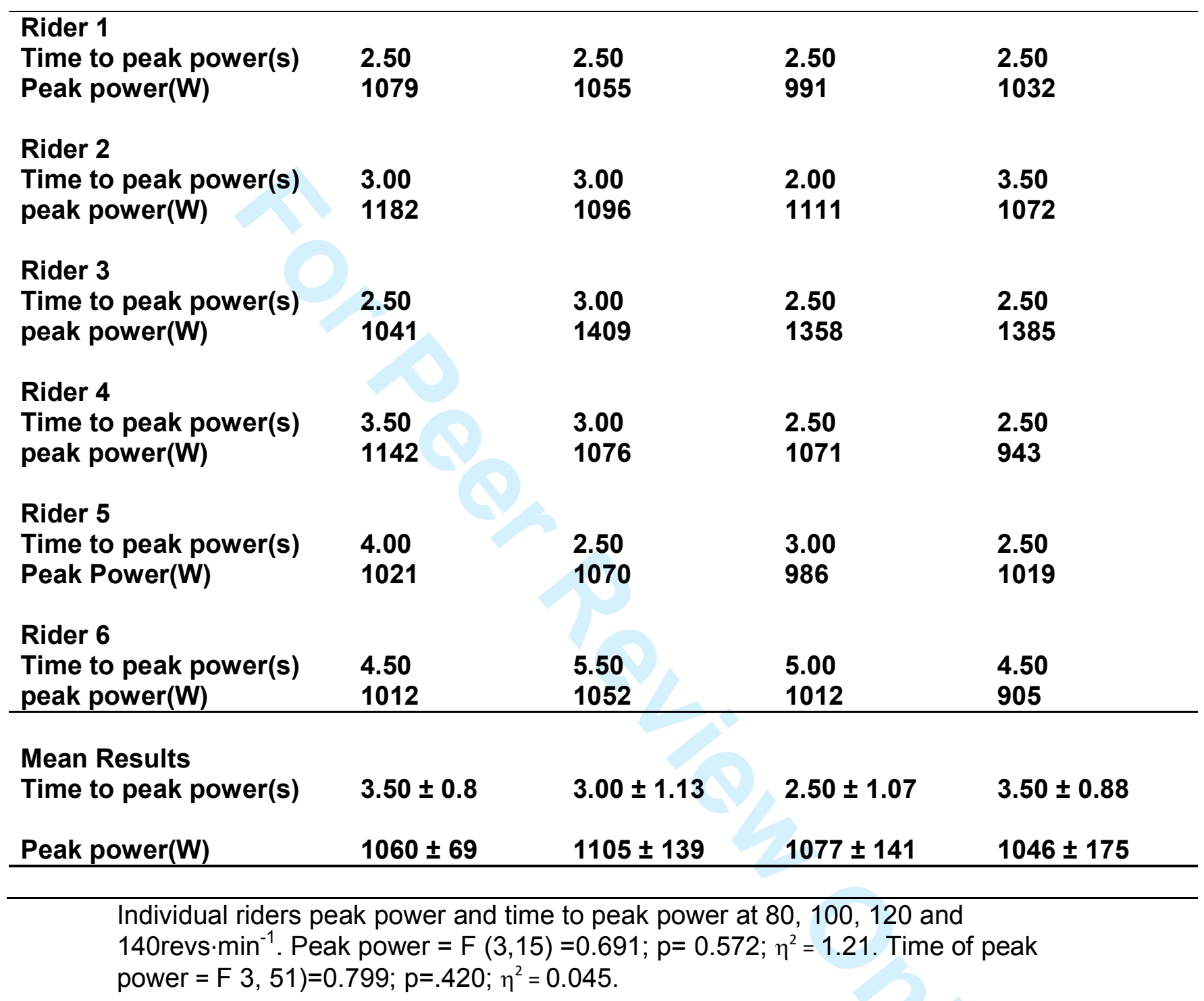




\section{Figure 1}

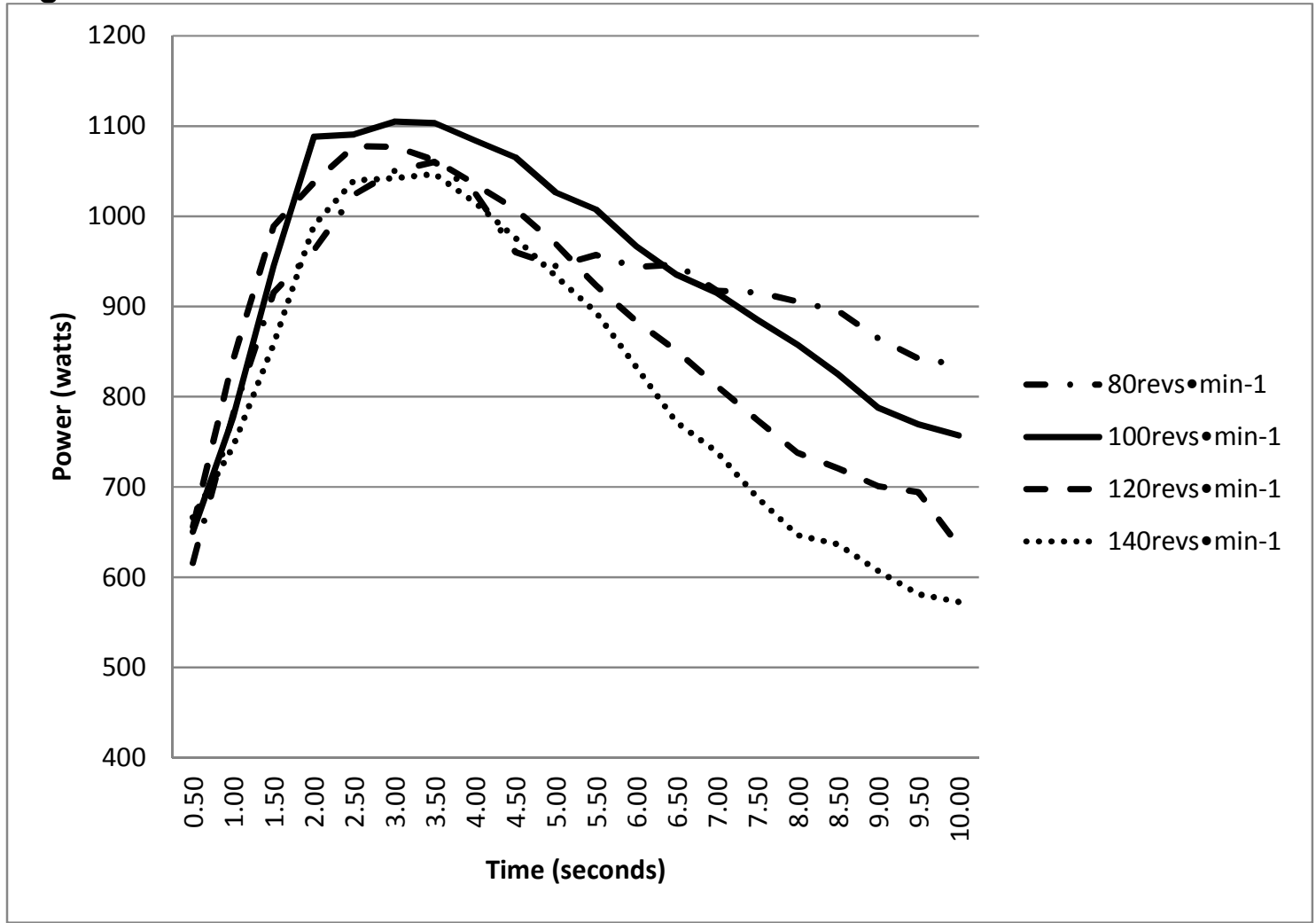

Results from 10 seconds sprint on a cycle ergometer were the cadence was restricted to $80,100,120$ and $140 \mathrm{revs} \cdot \mathrm{min}^{-1}$. Graph shows mean results for all six riders' peak power and power development 